\title{
Application of Moment of Momentum Theorem in Tie the Knot with A Hoop and A Chain
}

\author{
Zheng Shanshan ${ }^{1, a}$, Zheng Bin ${ }^{2, b}$, Qu Shuying ${ }^{3, c}$, Hao Bing ${ }^{4, d}$ \\ 1,2,3,4 Yantai University, Department of Civil Engineering, Yan Tai, China \\ *azssluckyq@163.com, bbinge199088@vip.qq.com, 'qsy_qu@163.com, d1678180660@qq.com
}

\begin{abstract}
Keywords: Magic, Moment of Momentum, Moment of Inertia, Angular Displacement, Angular Speed Abstract. The reason why magic is mysterious is because the masters have sufficient application and grasp of various kinds of scientific principles. Here, take "tie the knot" magic for example, carry out several experiments of a single hoop knotting with a chain, analyze the process of operation, movement and demonstration from the viewpoint of dynamics and kinematics to discuss the principle of mechanics, giving the comparison and analysis between all kinds of different technique of magic show and process of organic combination between hoops and chain, reveal its cognitive rule of scientific and deepen the understanding, cognizance and interesting in magic.
\end{abstract}

\section{Introduction of Magic-- “Tie the Knot”}

\section{Material}

Magic is wonderful, changeable, and has different subtleties fromeach other. It not only depends on the ingenious magic props, but also depends on the performers, manipulation for the props. In the "Tie the Knot" magic, the iron hoop and iron chain are independent and isolated fromeach other. Assembled as shown in Figure 1, the hoop and chain can rub against each other arbitrarily up and down, in this case, the hoop and chain are unable to tie a knot. But when relaxed instantly, the hoop drop down and knot with the chain.

The ingeniousness of this magic is that the hoop rotates based of the effect of external torque in the process of falling, entrance into the chain instantaneously, slip down to the chain tail, and achieve knotting with chain. In order to achieve the hoop

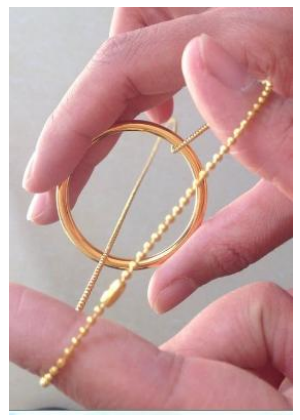
knotting with chain by rotating and sliding, the selection of magic props need to meet the following conditions:

(1) The material making hoop and chain has a bigger specific gravity;

(2) The surface of hoop and chain is smooth, and the coefficient of friction is very small;

(3) Every micro line of chain can be bended arbitrarily and freely, and the bending strength of chain is low.

\section{Sizes and Specifications of Components}

(1) The length of horizontal segment of iron chain is greater than the diameter of hoop and is about $5 \mathrm{~cm}$, so the hoop and chain can be in touch.

(2) When the rotation angular displacement angle of hoop is larger than $100^{\circ}$, the chain below the hoop must be long enough for knotting and slipping down.
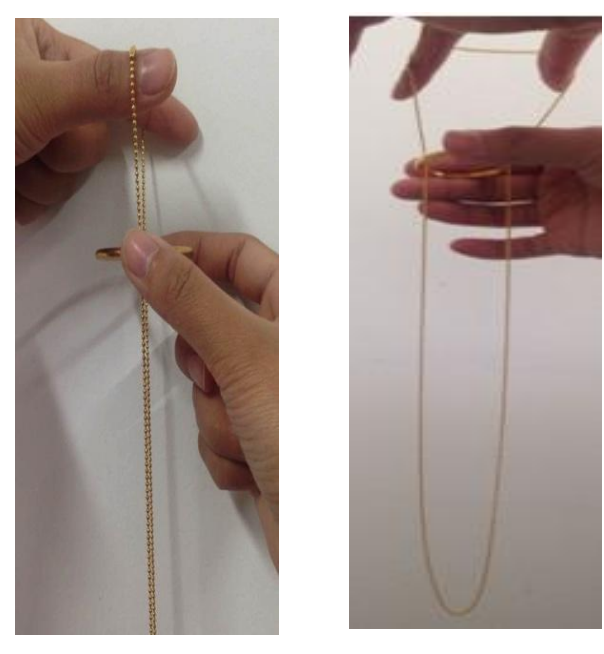

Figure2. TTK peformancı Figure3. TTK peformance ? 


\section{A single hoop knotting with a chain}

\section{Description of Magic Performance 1}

In performance 1, as can be seen in Figure 2, “Tie the Knot" (TTK) magic shows the iron hoop is restrained by right index finger and thumb to keep the level state naturally, the middle finger naturally bend and protrude, the key is to apply the techniques of hand in the process of the hoop instantly knotting with the chain. As seen in Figure 2, at the moment when the hoop collides with the middle finger in the process of falling, three continuous processes will be completed:

(1) The iron hoop freely and translational falls displacement h1;

(2) At the collision moment: the iron chain obtains a certain moment of momentum $\mathrm{J} \omega$;

(3) The iron hoop gets an initial angular speed $\omega$ to rotate; synchronously it falls vertically, and achieves knotting with the iron chain [3].

Now, let' $\mathrm{s}$ analyse these three stages separately, as shown in Figure 4.

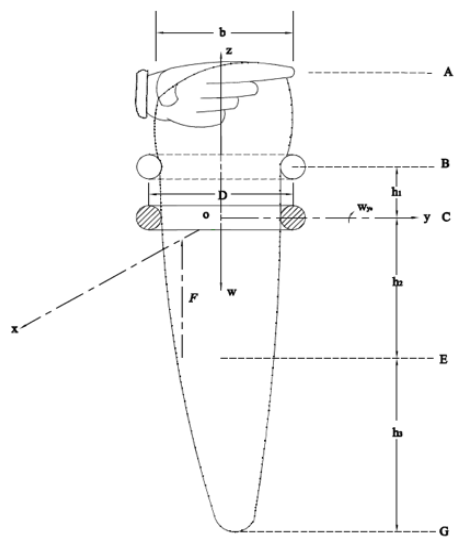

Figure 4. dynamic analysis

Stage 1: the iron hoop transnational falls with a displacement h1, due to gravity [4], the speed of hoop before collides with the middle finger can be calculated as:

$v=\sqrt{2 g h_{1}}$

Stage 2: The iron hoop has a collision with the projecting middle figure. The falling displacement can be ignored due to the extremely short time. In this stage, the iron chain obtains a certain moment of momentum and a certain initial angular speed. Known from the analysis of moment of momentum theorem [5], there is a certain relationship between the initial angular speed $\omega$ and impulse $S$ of the iron hoop, diameter of the iron hoop $\mathrm{D}$ and rotational inertia $\mathbf{J}$ of the iron hoop. In the process of collision, the impulse moment of the iron hoop equals to the moment of momentum of the iron hoop.

$S \times\left(\frac{D}{2}\right)=J \omega$

So for the angular speed of the iron hoop at the beginning of the stage 3 we have:

$\omega=\frac{S \times D}{2 J}$

Stage 3: During this falling process, the iron hoop with the initial angular speed, achieves a perfect knotting after it reached the angular displacement of $100^{\circ}$.

\section{Description of Magic Performance 2}

In performance 1, the iron hoop gains enough moment of momentum and initial angular speed through a collision, and it has a large enough angular displacement to make the hoop tie a knot with the chain. Of course, if there is no collision, we could have other method to achieve knotting between the hoop and the chain as well $[6,7]$.

We make a change to make the iron hoop gain enough moment of momentum to the rotation of the more than $100^{\circ}$, eventually it reaches the same result as the one before--the hoop knot with the chain.

The differences between Figure 2 and 3 are: the iron hoop is restrained by right index finger, middle finger and thumb to keep the level state naturally; and the direction of stretched chain is parallel to the direction of the right

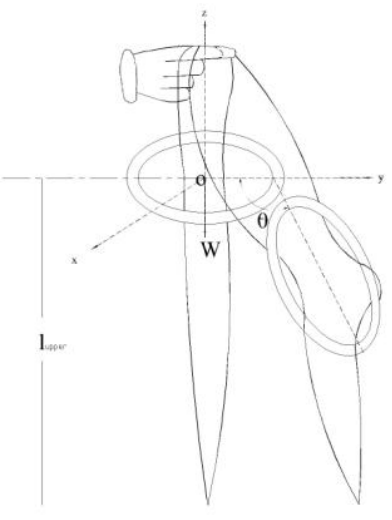

Figure 5. Mechanical analysis thumb. 
Figure 3 shows the initial preparation of the knotting process. Once the hoop is released, it will instantly set into the chain. The key point is the use of hand techniques, at the moment when a hoop falls, first relax your thumb naturally and make the hoop do the fixed axis rotation around the middle finger which is not relaxed (since it's a very short time, the falling displacement of the hoop in the no-relaxation side during this process can be neglected), rotational angular displacement is larger than $100^{\circ}$. Then the hoop disaffiliated from middle finger with a vertical downward accelerating translation, finishing the knot with chain eventually (Figure 5). The size of the
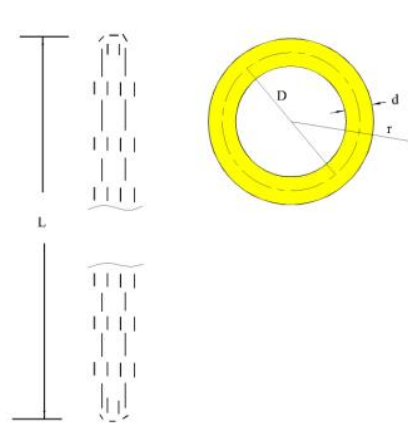
iron hoop and iron chain as shown in Figure 6.

In the falling process of the iron hoop fixed-axis rotation, the speed of iron hoop: $v=\sqrt{2 g r \sin \theta}$

Figure 6: Sizes of hoop and chain

In stage of rotation, the iron hoop gains moment of momentum and a certain angular speed at the same time, according to the moment of momentum theorem: $m v \times\left(\frac{D}{2}\right)=J \omega$

The angular speed of the iron hoop:

$\omega=\frac{m v D}{2 J}$

In the whole process of the hoop' s rotation, the displacement in vertical direction and the friction between the iron hoop and train is ignored due to the extremely short role time. Analyzing this process according to Figure 5, by the application of conservation of energy theorem [8]:

$$
\left(2 l-D-2 l_{\mathrm{wper}} \times m_{0} g r \times(1+\sin \theta)=m g r \cos \theta\right.
$$

When $\theta$ is more than $100^{\circ}$, the iron hoop and chain will complete a set of knot, then the hoop drops down with freely translation. The key to success for knotting is that the angular displacement $\theta$ of the iron hoop is larger than $100^{\circ}$. So how should we select materials and size of the magic props, in order to guarantee the completion of the magic smoothly?

Here we need to calculate and comparatively analysis the selection of hoop and chain parameters. First, calculation according to the known size of the hoop and chain in this article, according to conservation of energy theorem:

$(2 l-50-2 \times 40) \times m_{o} g r \times\left(1+\sin 100^{\circ}\right)=m g r \times \cos 100^{\circ}$

Get the maximum length of the chain that guarantees the chain knotting with the hoop. In the process of the iron hoop translational falling, gravity and frictional resistance of chain affect movement of hoop; the equations of motion $[9,10]$ for the hoop can be used to calculate the minimum length of the chain:

$m g-2 f \times 2 m_{0} \times\left(l_{\text {bower }}-y(t)\right) \times g=-m \ddot{y}(t)$

We need to analyze and compare the parameters of the iron hoop. As can be seen from the formula (2), the material with larger mass, bigger moment of inertia, smoother surface and lower friction factor can be knotted. Other materials like plastic, aluminium or wood, is difficult to knot with chain.

Table 1. Parameters for Different Types of Material

\begin{tabular}{cccccc}
\hline & $\rho_{m m}$ & $D_{m m}$ & $d_{m m}$ & $m_{g}$ & $\theta$ \\
\hline 1 & 7.93 & 70 & 4 & 21.9 & $135 \sim 140$ \\
2 & 7.70 & 70 & 4 & 21.3 & $130 \sim 136$ \\
& & 70 & 1.6 & 3.4 & 100 \\
3 & 4.50 & 60 & 4 & 10.6 & $125 \sim 135$ \\
4 & 2.60 & 50 & 4 & 5.13 & $86 \sim 100$ \\
\hline
\end{tabular}


Based on the table above, friction factor of the materials selected are relatively low. Because heavier material is easy to get a larger angular displacement and achieve set of knot with chain, and the lighter material gets the smaller angular displacement, and the probability of completing performance is low.

\section{Conclusion}

"Tie the Knot" magic described from two single hoops and chains junction, two hoops overlapping sets of nodes, two hoops dislocation superimposed set of nodes and three or more hoops tacking, it' s process and results also were analyzed in this paper. Reach the following conclusion:

3.1. The length of chain, the size of the hoop and the proportion of hoop are important factors that influence the magic is success or not, only within the scope of possible values can ensure the success of magic.

3.2. By exploring the scientific principles of magic, a magic phenomenon can be achieved by a variety of magic tricks to explore the experiment in two rings and chain.

3.3. Magic innovates constantly in exploring, it enriches people's lives and provides a steady stream of power to social progress and human development.

\section{Acknowledgement}

This work is supported by Higher Education Reform in Shandong Province Project (SD2012024) and Graduate Innovative Education Founds of Shandong Province (Grant No. SDYY11194). Special thanks to Zhuang Biaozhong for his direct contribution on the specific works.

\section{References}

[1] Lu Chen, "effect of Magic consciousness on the development of magic" ,China Acrobatics and Magic, No.3, pp.56 57, 2009

[2] Lu Yunting, “The Games Between Magic and Math: The Aesthetic Dimension of Art Soccer” , Journal of Sports and Science, Vol.34, No.4, pp.39 47, 2013

[3] Zhuang Biaozhong, Sun Chengqi, Wu Lixiang, “The third dynamic analysis of magic --Two or More Iron Hoops Knotting with Iron Chain” , Mechanics in Engineering, Vol.31, No.3, pp.108 109, 2009

[4] Qiao Hongzhou, “Theoretical Mechanics” , Beijing: China Building Industry Press, pp.204 261, 2009

[5] Zhou Yanbo, “Theoretical mechanics course” , Beijing: Higher Education Press, pp.150 210, 2009

[6] Qu Shuying, Zheng Shanshan, "Construction of Experiment Teaching Demonstration Centre with Interests Driven, Independent Experiments, and Exploratory Innovation ” , BIJ, Vol.10, No.20, pp.12032 12036, 2014

[7] Lin Gongshi, "selecting project of original innovation scientific research" , Science of Science and Management, pp.36 39, 2004

[8] Theoretical Mechanics Teaching and Research Section of Harbin Industrial University, “Theoretical Mechanics ( II )” , Beijing: Higher Education Press, 2009

[9] Ray Clough, “structural dynamics” , Beijing: Higher Education Press, No.3, pp.13 15, 1979

[10]Liu Hezhi, Zhang Long, “simple analysis of the magic hoop falling stress process” , science and technology information, No.7, pp.253, 2012 
